\section{A new type of transient high-energy source in the direction of the Galactic Centre}

\author{
C. Kouveliotou* $\dagger$, J. van Paradijs $\ddagger \S$, G. J. Fishman $\nmid$, \\ M. S. Brigss\$, J. Kommers\|, B. A. Harmont, \\ C. A. Meegant \& W. H. G. Lewin
}

* Universities Space Research Association, 4950 Corporate Drive, Suite 100, Huntsville, Alabama 35806, USA

† NASA Marshall Space Flight Center, ES-84, Huntsville, Alabama 35812. USA

‡ Astronomical Institute "Anton Pannekoek" and Center for High-Energy Astrophysics, Kruislaan 403, 1098 SJ Amsterdam, The Netherlands $\$$ Physics Department, University of Alabama in Huntsville, Huntsville, Alabama 35899. USA

Department of Physics, Center for Space Research, MIT 37-627, Cambridge, Massachusetts 02139, USA

Sources of high-energy ( $>20 \mathrm{keV}$ ) bursts fall into two distinct types: the non-repeating $\gamma$-ray bursters ${ }^{\prime}$, several thousand of which have been detected but whose origin remains unknown, and the soft $\gamma$-ray repeaters (SGRs), of which there are only three ${ }^{2}$. The SGRs are known to be associated with supernova remnants, suggesting that the burst events most probably originate from young neutron stars ${ }^{3}$. Here we report the detection of a third type of transient high-energy source. On 2 December 1995 , we observed the onset of a sequence of hard X-ray bursts from a direction close to that of the Galactic Centre". The interval between bursts was initially several minutes, but after two days, the burst rate had dropped to about one per hour and has been largely unchanged since then. More than 1,000 bursts have now been detected, with remarkably similar light curves and intensities; this behaviour is unprecendented among transient $X$-ray and $\gamma$-ray sources. We suggest that the origin of these bursts might be related to the spasmodic accretion of material onto a neutron star.

Visual inspection of the X-ray intensity records of the eight Large Area Detectors (LADs) of BATSE ${ }^{5}$ showed that bursts of hard X-rays were emitted in rapid succession from a source in the general direction of the Galactic Centre beginning 1995 December $212: 17$ uT (Fig. 1). The bursts came at intervals of about 5 minutes; they lasted for typically 30 seconds and reached a peak intensity of $\sim 300$ countss ' 1 in the $25-60 \mathrm{keV}$ energy band. Spectral analysis of the events showed significant emission to $\sim 60 \mathrm{keV}$ in virtually all bursts; in $\sim 17 \%$ of them, emission was detected to about $75 \mathrm{keV}$. The outbursts were also registered by the Spectroscopy Detectors ${ }^{5}$ which look into the same sky directions as the LADs and are sensitive down to $\sim 10 \mathrm{keV}$. The bursts usually show a clear single peak; in many cases a tail of extended cmission can be seen. The burst which we could unambiguously identify as the first in this sequence of rapid hard $\mathrm{X}$-ray bursts occurred on December 2 ()8:22 UT. Between December 2 17:(K) and 20:00 UT, the burst intervals clustered around (172 \pm 15$) \mathrm{s}$. After 20:00 uT the intervals became more erratic and longer. Figure 2 shows the behaviour of the burst interval evolution during the first 4 days of emission. Between 4 and 16 December the burst detection rate remained remarkably constant at an average of 18 events per day (about one-third of the bursts were missed because of data gaps).

We determined the location of the individual events from the relative strengths of the signals detected in different BATSE detectors, using an algorithm that includes a detailed description of the energy/angular detector response and the contribution from $\mathrm{X}$-rays scattered off the Earth atmosphere'. Initially, the bursts were seen in two detectors only; to improve the constraints on the location, the Compton Gamma Ray Observatory (CGRO) was reoriented on December 8 00:57 uT, after which the events were registered by four detectors. The BATSE locations combined with (1) Earth occultation constraints of the source, (2) a galactic longitude constraint from observations with the Oriented Scintillation Spectroscopy Experiment (OSSE) on $\mathrm{CGRO}^{7}$, and (3) Interplanetary Network (IPN) triangulation results ${ }^{\times, 9}$, produced an $\sim 24^{\prime} \times 6^{\prime}$ error box centred on right ascension $17 \mathrm{~h} 44 \mathrm{~min} 28 \mathrm{~s}$, declination -28 45.0' (equinox 2000.0). A 3-arcmin error box recently ${ }^{10}$ obtained with the X-Ray Timing Explorer (XTE) lies entirely within the IPN location. This error box does not contain known bright X-ray sources".

As part of a current project ${ }^{2}$ we made a comprehensive search for events in the $20-60 \mathrm{keV}$ energy range in archival BATSE data, covering the period 13 January 1993 to 24 December $1993(345$
FIG. 1 The BATSE (25-60 keV) intensity records obtained during three consecutive orbits of CGRO on 2 December 1995 between 13:50 and 17:30 uT; the data are displayed with a time resolution of $4 \mathrm{~s}$. (The time scale starts at 00:00 ut on 2 December 1995).
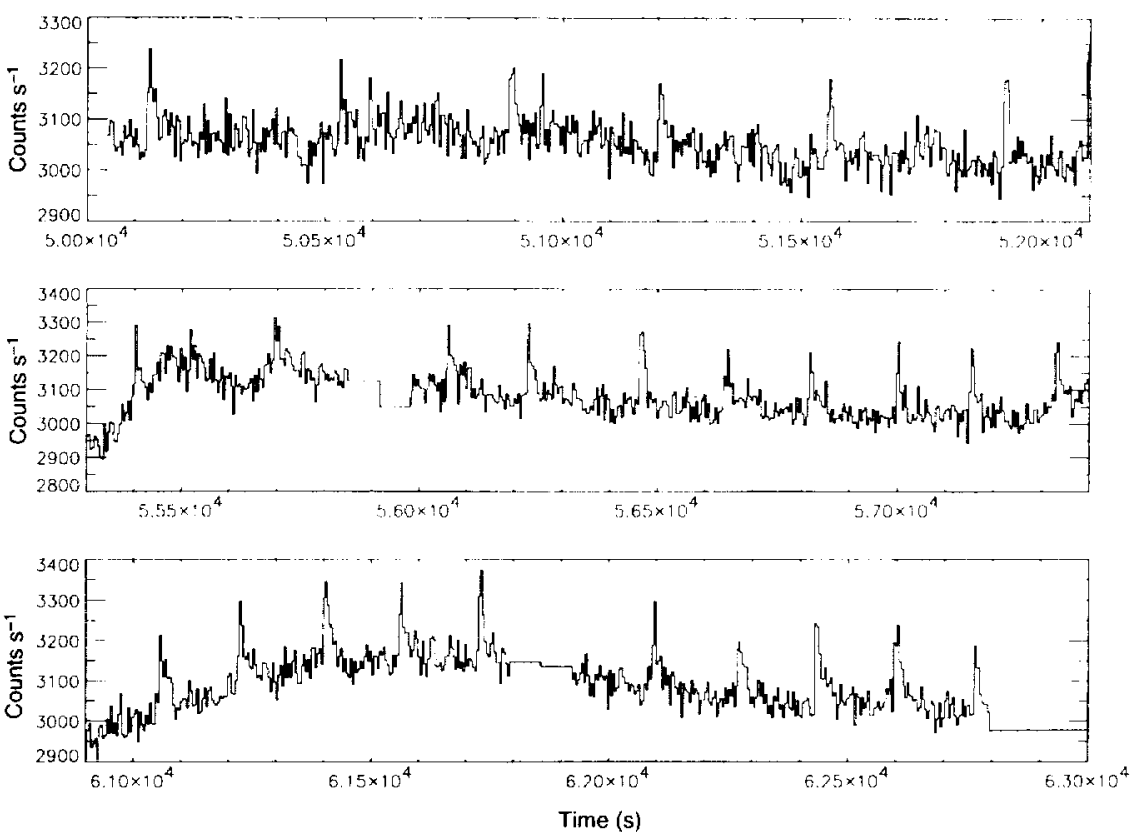
days). No activity which is unambiguously associated with the new transient was found.

In the following description of the properties of the hard X-ray bursts we limit ourselves to the 143 events detected during the first 4 days of burst activity, 134 of which have complete data. The durations of the bursts have been estimated from visual inspection of the X-ray intensity curves $(25-60 \mathrm{keV}$, with $2-\mathrm{s}$ time resolution). They range between $\sim 6$ and $\sim 100$ s (estimated accuracy $\pm 4 \mathrm{~s}$ ), with the majority of the events between 10 and $30 \mathrm{~s}$. Based on the count rates (and upper limits to them) in the CONT data ${ }^{2}$ energy channels, we have made spectral fits, using several spectral models. Because of the limited statistics of the signals of individual events, we cannot make a significant distinction between a powerlaw fit (describing non-thermal emission) and thermal models, such as a black-body and thermal bremsstrahlung. The spectral parameters for the different bursts are rather similar; photon indices $x$ range between -4.6 and -5.5 with an average value of -5.0 (average reduced $\chi^{2}$ value is 1.73 (7 d.o.f.)). The typical $k T$ values are $\sim 5 \mathrm{keV}$ for black-body fits and $\sim 9 \mathrm{keV}$ for thermal bremsstrahlung fits.

Using the power-law spectral fits we determined for each burst the $(25-60 \mathrm{keV})$ peak flux, $F_{p}$, and the fluence $S$. The fits were made in order to convert count rates to fluxes and fluences. No particular physical meaning should be attached to the fit results, except as an indication of the hardness of the burst spectra. With few exceptions, the peak fluxes $(25-60 \mathrm{keV})$ are confined to a fairly narrow interval. The average $\vec{F}_{\mathrm{n}}=(2.8 \pm 0.7) \times$ $10{ }^{*} \mathrm{erg} \mathrm{cm} \mathrm{s}^{\prime}(1 \sigma)$. This may suggest that some limiting process is involved in the burst production. For a distance of $7.5 \mathrm{kpc}$ (suggested by the small angular distance of the burst source to the Galactic Centre) the average $(25-60 \mathrm{keV})$ peak luminosity is $2 \times 10^{3 \times} \mathrm{ergs}$. Although the bolometric luminosity depends on an uncertain extrapolation of the spectrum to lower energies, it is of interest to note that this value is of the same order as the Eddington luminosity of an object of one solar mass (assuming solar composition for the accreted material).

We have computed the integral hardness ratio per event, that is, the ratio of total counts in the $33-60 \mathrm{keV}$ and $25-33 \mathrm{keV}$ bands. The event hardness remains remarkably constant, with an average of $1.20 \pm 0.24$. Unlike classical $;$-ray bursters (GRBs), there is no dependence of hardness on duration ${ }^{13}$. Preliminary comparisons of the hardness ratios as measured for the first half of the counts in a burst (integrated over all energies) with that of the second half, find no evidence for spectral evolution during the bursts, similar to the bursts from soft "-raly repeaters (SGRs).

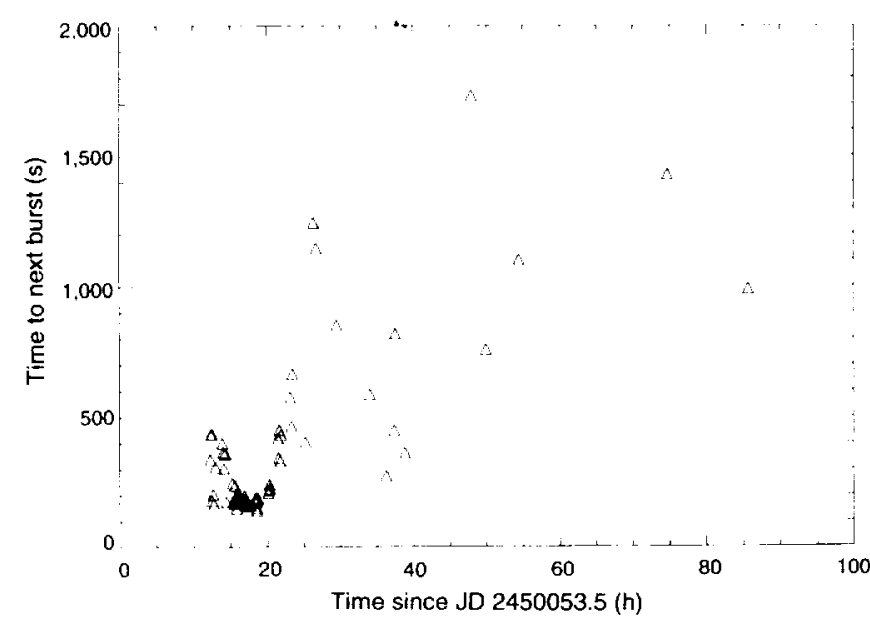

FIG. 2 Evolution of the intervals between bursts, from 14:00 uT on 2 December 1995 onwards. Note the very regular spacing of $172 \mathrm{~s}$ between bursts between 4 and 6 hours after the start of this sequence, and the subsequent increase and loss of regularity of the intervals.

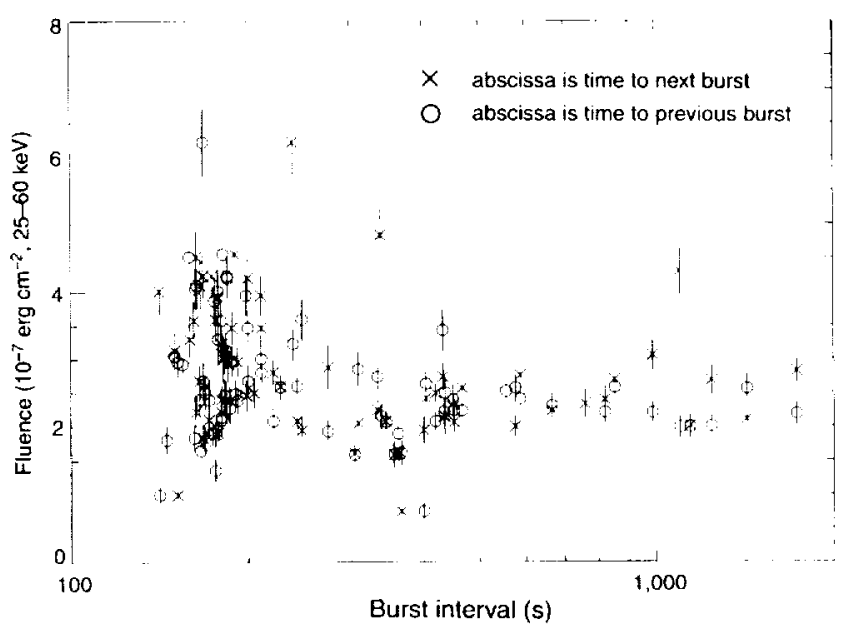

FIG. 3 Relation between the burst fluence and the time interval since the previous burst (crosses) and the time interval till the next burst (circles).

The burst fluences range between $1.7 \times 10^{7}$ and $6.8 \times$ $10{ }^{7} \mathrm{erg} \mathrm{cm}^{2}$; the average fluence $\bar{S}=(2.7 \pm 0.9) \times 10^{7} \mathrm{crg} \mathrm{cm}^{2}$. In Fig 3 we show the relation between the burst flucnce and the time interval before $(\Delta t)$ and the interval after $\left(\Delta t^{+}\right)$the burst. The burst fluences do not show an obvious correlation with either time interval; for long intervals they appear to be almost constant.

The bursts we have detected are unlike those from any known source. They are very different from GRBs, which show no evidence of fast repetition and whose spectra are much harder (the bulk of their energy is above $100 \mathrm{keV}$; ref. 14). Their rapid recurrence is reminiscent of the rapidly repetitive type II bursts from the Rapid Burster ${ }^{15}$, whose nature still remains a mystery. However, the error box of the new burst source excludes the Rapid Burster.

With respect to their repetition and their spectra, the bursts we detected are similar to the events that have been observed from the three SGRs ${ }^{2,3.16}$. However, they are very different in that they last several hundred times longer than SGR events (with the exception of two bursts from SGR0525 - 26 which lasted 3.5 and $8.5 \mathrm{~s}$, respectively ${ }^{17}$ ). We can exclude the possibility that the hard X-ray bursts originate from the position ${ }^{18}$ of SGR1806-20 because one of them was observed when this source was occulted by the Earth.

To explain high-energy transients, three types of energy source have been proposed: (1) thermonuclear flashes in matter accreted on the surface of a neutron star; (2) unstable accretion onto a compact star; (3) internal energy sources of a neutron star (rotational and magnetic energy).

We can exclude the possibility that these bursts are caused by thermonuclear flashes on accreting neutron stars with low magnetic fields, as these sources produce the relatively well understood type I X-ray bursts ${ }^{15}$ whose spectra are described by blackbody radiation, with temperature $k T_{\mathrm{Bg}}$ generally below $2 \mathrm{keV}$, and which show distinct cooling during burst decay. Thermonuclear flashes on the surface of a strongly magnetized neutron star (if they occur at all) have becn proposed as a model for GRBs ${ }^{14}$ and may have much harder spectra than type I bursts. However, such flashes should have very long recurrence times.

Independent of detailed thermonuclear flash models, during the period of rapid burst repetition the required replenishment of burnt fuel (through accretion onto the neutron star) would lead to an accretion flux of the order of several times $10^{4} \mathrm{erg} \mathrm{cm}^{2} \mathrm{~s}{ }^{1}$, that is, one would expect a very bright $X$-ray source. On the basis of the BATSE occultation data, which provides an upper limit of $\sim 10^{-9} \mathrm{erg} \mathrm{cm}^{-2} \mathrm{~s}$ to the persistent flux, we can exclude the possibility that this persistent accretion power is emitted as X-rays with energies in excess of $\sim 10 \mathrm{keV}$. For the ratio of persistent flux 
to time-averaged burst flux ( $\alpha$, ref. 15 ), as measured in the BATSE energy range, we find a $3 \sigma$ upper limit of $\leqslant 4$. These results virtually rule out the possibility that the bursts are caused by thermonuclear flashes on neutron stars.

We consider accretion instabilities a viable model for the bursts from the Galactic Centre region. If this model is correct, it implies that the source of hard X-ray bursts is part of a binary system. In that case, it is likely that the matter is accreted onto the compact object in spasmodic fashion after storage in an accretion disk. Disk storage has been suggested as a model for the type Il bursts from the Rapid Burster ${ }^{20}$; however, the hard X-ray bursts that we observed differ from the type II bursts of the Rapid Burster by their much harder spectra, and by not following the relaxation oscillator recurrence pattern shown by that source. This does not, however, exclude the possibility that the bursts reported here are caused by some accretion instability. In particular, the almost regular intervals between the bursts are much more reminiscent of the Rapid Burster than of the SGR sources, pointing to a triggering mechanism similar to the one for type II bursts rather than to SGR bursts.

According to the SGR model of Thomson and Duncan ${ }^{21}$, hard $X$-ray events may originate from strongly magnetized neutron stars (magnetars) through the release of magnetic field energy, which leads to the formation of a pair plasma confined by the magnetosphere. The duration of the events is determined by the diffusion time of the plasma, which is proportional to the inverse square of the magnetic field strength. Although we favour an accretion instability origin for the bursts, we believe that magnetar models cannot be excluded.

If the bursts reported here are due to accretion instabilities, which is likely, they are by definition ${ }^{15}$ type II bursts. The question then remains why are they so different from those of the Rapid Burster. The answer could lie with the compact object which may be a neutron star with a much higher magnetic field than the Rapid Burster.

Note added in proof: Since this Letter was submitted, a persistent source, GROJ1744 - 28, has been found ${ }^{22}$ and later identified as a binary X-ray pulsar ${ }^{23,24}$. The burster and the pulsar are the same object $^{25}$.

Received 3 January; accepted 5 February 1996.

1. Fishman, G. J. \& Meegan, C. A. A. Rev. Astr. Astrophys. 33, 415-458 (1995)

2. Norris, J. P., Hert, P., Wood, K. \& Kouveliotou, C. Astrophys. J. 3e6, 240252 (1991)

3. Kulkami, S. R., Frail, D. A., Kassim, N. E., Murakami, T. \& Vasisht, G. Nature 3es, 129-131 (1994).

4. Fishman, G. J. et al. IAU Circ. No. 6272 (1995).

5. Fishman, G. J. et al. in Proc. Gamma Ray Obsenatory Science Workshop (ed. Johnson, W. N.) 39-50 (NASA, Goddard Space Flight Center, Greenbelt, MD, 1989)

6. Pendieton, G. et al. Astrophys. J. (in the press).

7. Kurfess, J. D. et al. IAU Circ. No. 6278 (1995).

8. Hurley, K. et ai, IAU Circ. No. 6276 (1995).

9. Hurley, K. et al IAU Circ. No. 6286 1996).

10. Swank, J. IAU Circ. No. 6291 (1996).

11. Van Paradijs, J. in X-ray Binaries (eds Lewin, W. H. G. et al.) 536-577 (Cambridge Univ. Press, 1995).

12. Kommers, J. et al. in Proc. 3rd Huntsville Gamma Ray Burst Workshop (eds Kouveliotou, C. Bnggs, M. S. \& Fishman, G. J.) (in the press).

13. Kowveliotou, C. et al. Astrophys. J. 413, L101-L104 (1993)

14. Band, D. et al. Astrophys. J. 413, 281.292 (1993).

15. Lewin, W. H. G., Van Paradijs, J. \& Taam, R. E. in X-ray Binaries (ed. Lewin, W. H. G. et al.) 175 232 (Cambridge Univ. Press, 1995).

16. Kouveliotou, C. et al. Nature 362, 728-730 (1993).

17. Mazets, E. P. et at. Astrophys. Space Sci $80,3-143$ (1981)

18. Van Kerkwitk, M. H. et at Astrophys. J 40, L61-L64 (1995)

19. Woosley. S. E. \& Wallace, R. K. Astrophys. J. 238, 716-732 (1982)

20. Spruit, H. C. \& Taam, R. E. Astrophys. 1. 402, 593-604 (1993).

21. Thomson, C. \& Duncan, R. G. Astrophys. J. (in the press).

22. Paciesas, W. S., Harmon, B. A., Fishman, G. J., Zhang, S. N. \& Robinson, C. R. IAU Circ. No. 6284 (1996).

23. Finger, M. H., Wilson, R. B., Harmon, B. A., Hagedon, K. \& Prince, I. A. IAU Circ. No. 6285 (1996).

24. Finger, M. H. Wilson, R. B. \& van Paradjis, J IAU Circ No. 6286 (1996).

25. Kouveliotou, C. et al. IAU Circ. No. 6286 (1996)

ACKNOWLEDGEMENTS. J.V.P., C.K. and W.H.G.L. were supported by NASA; J.M.K. was supported by a National Science Foundation graduate research fellowship. 
